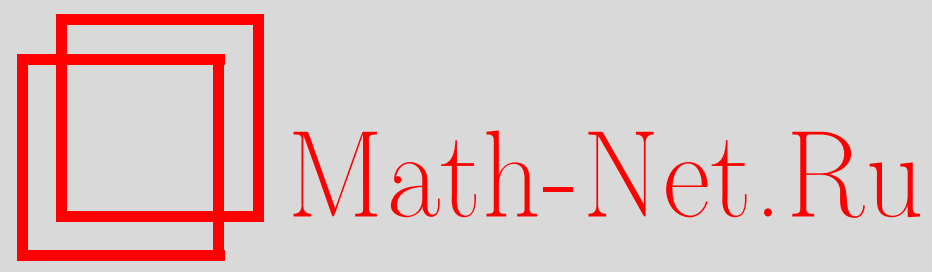

О. А. Логачев, С. В. Смышляев, В. В. Ященко, $\rho$ уравновешенные булевы функции, Дискрет. матем., 2012, том 24, выпуск 2, 154-159

DOI: https://doi.org/10.4213/dm1191

Использование Общероссийского математического портала Math-Net.Ru подразумевает, что вы прочитали и согласны с пользовательским соглашением http://www.mathnet.ru/rus/agreement

Параметры загрузки:

IP: 54.237 .206 .68

26 апреля 2023 г., $15: 23: 33$ 
УДК 519.2

\title{
$\rho$-уравновешенные булевы функции
}

\author{
() 2012 г. О. А. Логачев, С. В. Смышляев, В. В. Ященко
}

\begin{abstract}
Вводится параметризованное семейство свойств булевых функций, обобщающих свойства уравновешенности и совершенной уравновешенности. При этом свойства уравновешенности и совершенной уравновешенности входят в это семейство при некоторых крайних значениях параметра. Изучается структура соответствующего семейства множеств булевых функций, а также свойства функций из этих множеств.

Работа выполнена при поддержке Российского фонда фундаментальных исследований, гранты 09-01-00653-а и 10-01-00475-а.
\end{abstract}

\section{1. Введение}

Одним из важнейших свойств кодирующих устройств, построенных с помощью регистров сдвига и булевых функций, является возможность получения на выходе произвольных двоичных последовательностей. Данное свойство, как было показано Сумароковым ([7]), эквивалентно понятию совершенной уравновешенности булевых функций.

В настоящей работе вводится и исследуется свойство $\rho$-уравновешенности булевых функций для фиксированного каркаса $\rho$, обобщающее, в определенном смысле, свойство совершенной уравновешенности. Формулируется и доказывается критерий $\rho$-уравновешенности в терминах коэффициентов Уолша-Адамара, важный для получения соотношений между коэффициентами Уолша-Адамара совершенно уравновешенных булевых функций.

Доказывается утверждение о том, что множество $\rho$-уравновешенных булевых функций при определенном классе каркасов $\rho$ строго вложено в множество совершенно уравновешенных функций. Данное утверждение является обобщением представленного в [2] результата, опровергающего полученный Голичем в [1] критерий совершенной уравновешенности.

\section{2. Определения и предварительные результаты}

Для любого натурального $n$ множество двоичных наборов длины $n$ будем обозначать через $V_{n}=\{0,1\}^{n}$; множество булевых функций от $n$ переменных - через $\mathscr{F}_{n}$.

Определение 1 ([4]). Преобразованием Уолша-Адамара булевой функции $f$ называется целочисленная функция на $V_{n}$, определяемая равенством

$$
W_{f}(\mathbf{u})=\sum_{\mathbf{x} \in V_{n}}(-1)^{f(\mathbf{x}) \oplus\langle\mathbf{x}, \mathbf{u}\rangle}
$$


(суммирование производится в действительной области). Для каждого $\mathbf{u} \in V_{n}$ значение $W_{f}(\mathbf{u})$ называется коэффициентом Уолша-Адамара.

Теорема 1 ([4]). Для преобразования Уолша-Адамара справедлива формула обращения:

$$
(-1)^{f(\mathbf{x})}=2^{-n} \sum_{\mathbf{u} \in V_{n}} W_{f}(\mathbf{u})(-1)^{\langle\mathbf{x}, \mathbf{u}\rangle} .
$$

Обозначим через $\rho$ набор целых положительных чисел $\rho=\left\{k ; d_{1}, d_{2}, \ldots, d_{k}\right\}$, причем $d_{1}=1, d_{i+1}>d_{i}$ для всех $i=1,2, \ldots, k-1$.

Пусть теперь $\mathbf{y} \in V_{\infty}-$ бесконечная двоичная последовательность, $\mathbf{y}=\left(y_{1}, y_{2}, \ldots\right)$. Определим поднабор $\mathbf{y}_{\rho} \in V_{k}$ последовательности у равенством $\mathbf{y}_{\rho}=\left(y_{d_{1}}, y_{d_{2}}, \ldots, y_{d_{k}}\right)$.

Параметр $\rho$ поднабора $\mathbf{y}_{\rho}$ задает длину $\mathbf{y}_{\rho}$ и позиции последовательности $\mathbf{y}$, из которых выбираются элементы $\mathbf{y}_{\rho}$. Поэтому параметр $\rho$ будем называть каркасом поднабора $\mathbf{y}_{\rho}$.

Обозначим для функции $f \in \mathscr{F}_{n}$ и каркаса $\rho$ через $f_{\rho}$ следующее отображение из $V_{d_{k}+n-1}$ в $V_{k}$ :

$$
\begin{aligned}
& f_{\rho}\left(x_{1}, x_{2}, \ldots, x_{d_{k}+n-1}\right) \\
& \quad=\left(f\left(x_{1}, x_{2}, \ldots, x_{n}\right), f\left(x_{d_{2}}, x_{d_{2}+1}, \ldots, x_{d_{2}+n-1}\right), \ldots, f\left(x_{d_{k}}, x_{d_{k}+1}, \ldots, x_{d_{k}+n-1}\right)\right) .
\end{aligned}
$$

Определение 2. Функция $f \in \mathscr{F}_{n}$ называется $\rho$-уравновешенной, если отображение $f_{\rho}$ уравновешено; другими словами, если все поднаборы $\mathbf{y}_{\rho} \in V_{k}$ имеют равное число прообразов относительно отображения $f_{\rho}$.

Рассмотрим два каркаса

$$
\rho_{1}=\{1 ; 1\}, \quad \rho_{M}=\{M ; 1,2, \ldots, M\},
$$

где $M \in \mathbf{N}$. Очевидно, что $\rho_{1}$-уравновешенность булевых функций - это уравновешенность.

Определение 3. Булева функция $f \in \mathscr{F}_{n}$ называется совершенно уравновешенной, если она является $\rho_{M}$-уравновешенной при любом $M \in \mathbf{N}$.

Нетрудно показать, что данное определение является эквивалентным традиционному определению совершенной уравновешенности булевой функции в том виде, в котором оно представлено в $[1,5,6,7]$ и др.

\section{3. Основные результаты}

В [8] рассмотрено понятие сводимости свойств булевых отображений к свойствам координатных функций и показано, что свойство уравновешенности булевых отображений является сводимым. Из этого вытекает следующее утверждение.

Теорема 2. Функиия $f \in \mathscr{F}_{n}$ является $\rho$-уравновешенной тогда и только тогда, когда для любого ненулевого набора $\gamma=\left(\gamma^{(1)}, \gamma^{(2)}, \ldots, \gamma^{(k)}\right) \in V_{k}$ булева функиия $\sum_{\gamma} f_{\rho} \in \mathscr{F}_{d_{k}}+n-1$,

$$
\begin{aligned}
& \sum_{\gamma} f_{\rho}\left(x_{1}, x_{2}, \ldots, x_{d_{k}+n-1}\right)=\gamma^{(1)} f\left(x_{1}, x_{2}, \ldots, x_{n}\right) \\
& \oplus \gamma^{(2)} f\left(x_{d_{2}}, x_{d_{2}+1}, \ldots, x_{d_{2}+n-1}\right) \oplus \ldots \oplus \gamma^{(k)} f\left(x_{d_{k}}, x_{d_{k}+1}, \ldots, x_{d_{k}+n-1}\right),
\end{aligned}
$$

является уравновешенной. 
Введем на множестве каркасов отношение частичного порядка $\preccurlyeq$ следующим образом: если $\rho^{\prime}=\left\{k^{\prime} ; d_{1}^{\prime}, d_{2}^{\prime}, \ldots, d_{k^{\prime}}^{\prime}\right\}, \rho=\left\{k ; d_{1}, d_{2}, \ldots, d_{k}\right\}$, то $\rho^{\prime} \preccurlyeq \rho$ тогда и только тогда, когда $k^{\prime} \leqslant k$ и для любого $i=1,2, \ldots, k^{\prime}$ найдется $j(i) \in\left\{d_{1}, d_{2}, \ldots, d_{k}\right\}$ такое, что $d_{i}^{\prime}=d_{j(i)}$.

В качестве примера заметим, что для любого каркаса $\rho$ выполнено $\rho_{1} \preccurlyeq \rho$.

В этих обозначениях из теоремы 2 легко получить следующие утверждения.

Следствие 1. Функиия $f \in \mathscr{F}_{n}$ является $\rho$-уравновешенной тогда и только тогда, когда $f$ является $\rho^{\prime}$-уравновешенной для любого $\rho^{\prime} \preccurlyeq \rho$.

Следствие 2. Функиия $f \in \mathscr{F}_{n}$ является совершенно уравновешенной тогда и только тогда, когда она является $\rho$-уравновешенной для любого $\rho$.

Предположим теперь, что для каркаса $\rho$ найдется такое $i$, что $d_{i+1} \geqslant d_{i}+n$. В этом случае функция $\sum_{\gamma} f_{\rho}$ для любого набора $\gamma$ является суммой двух функций от непересекающихся переменных и, тем самым, является уравновешенной тогда и только тогда, когда хотя бы одно из слагаемых уравновешено. Очевидно при этом, что уравновешенность каждого слагаемого - это $\rho^{\prime}$-уравновешенность функции $f$ для некоторого $\rho^{\prime} \preccurlyeq \rho$. Тем самым, в дальнейшем достаточно рассматривать только такие каркасы $\rho$, для которых $d_{i+1}<d_{i}+n$ для любого $i=1,2, \ldots, k-1$. Аналогичными рассуждениями приходим к выводу, что при исследовании уравновешенности функций $\sum_{\gamma} f_{\rho}$ достаточно ограничиться такими наборами $\gamma$, при которых между любыми соседними слагаемыми обязательно есть зацепление, т.е. они имеют вид $f\left(x_{l}, x_{l+1}, \ldots, x_{l+n-1}\right) \oplus f\left(x_{m}, x_{m+1}, \ldots, x_{m+n-1}\right)$, где $m \leqslant l+n-1$. Кроме того, некоторые суммы $\sum_{\gamma} f_{\rho}$ и $\sum_{\gamma^{\prime}} f_{\rho}$ фактически будут задавать одну и ту же функцию после соответствующей перенумерации аргументов (сдвига номеров аргументов так, чтобы первой переменной была $\left.x_{1}\right)$.

Критерием $\rho$-уравновешенности функции $f \in \mathscr{F}_{n}$, который приведен в теореме 2 , является уравновешенность набора из $2^{k}-1$ функций вида $\sum_{\gamma} f_{\rho}$. Как показывают предыдущие рассуждения, на самом деле достаточно проверить уравновешенность меньшего числа функций вида $\sum_{\gamma} f_{\rho}$. При этом необходимо рассматривать каркасы $\rho^{\prime} \preccurlyeq \rho$ последовательно по мере увеличения сложности суммы, поскольку это позволит упрощать получаемые соотношения.

Таким образом, необходимо исследовать на уравновешенность булевы функции, которые представляются суммами вида

$$
\bigoplus_{i=1}^{m} f\left(x_{l_{i}}, x_{l_{i}+1}, \ldots, x_{l_{i}+n-1}\right)
$$

При этом набор номеров аргументов удовлетворяет следующим условиям:

(1) $\bigcup_{i=1}^{m}\left\{l_{i}, l_{i}+1, \ldots, l_{i}+n-1\right\}=\{1,2, \ldots, N\}$;

(2) $l_{i} \neq l_{j}$ для любых $i \neq j$;

(3) для любого $i \in\{2,3, \ldots, m\}$ найдется по крайней мере одно $j, j \neq i$, такое, что $l_{i} \in\left\{l_{j}, l_{j}+1, \ldots, l_{j}+n-1\right\}$.

Введем $N$ множеств пар индексов по следующему правилу:

$$
J_{k}=\left\{(i, s), i \in\{1,2, \ldots, m\}, s \in\{0,1, \ldots, n-1\} \mid l_{i}+s=k\right\} .
$$


Теорема 3. Булева функция (1) уравновешена тогда и только тогда, когда коэффициентьл Уолша-Адамара функции $f$ удовлетворяют следующему условию:

$$
\sum \prod_{i=1}^{m} W_{f}\left(\alpha^{(i, 0)}, \alpha^{(i, 1)}, \ldots, \alpha^{(i, n-1)}\right)=0
$$

где суммирование осуществляется по всем наборам $m n$ булевых переменных $\alpha^{(i, s)}$, $i \in\{1,2, \ldots, m\}, s \in\{0,1, \ldots, n-1\}$, которые удовлетворяют следующим $N$ линейным соотношениям:

$$
\bigoplus_{(i, s) \in J_{k}} \alpha^{(i, s)}=0, \quad k \in\{1,2, \ldots, N\}
$$

Доказательство. Функция (1) уравновешена тогда и только тогда, когда выполнено соотношение

$$
\sum_{\left(x_{1}, x_{2}, \ldots, x_{N}\right) \in V_{N}}(-1)^{\oplus_{i=1}^{m} f\left(x_{l_{i}}, \ldots, x_{l_{i}+n-1}\right)}=\sum_{\left(x_{1}, x_{2}, \ldots, x_{N}\right) \in V_{N}} \prod_{i=1}^{m}(-1)^{f\left(x_{l_{i}}, \ldots, x_{l_{i}+n-1}\right)}=0 .
$$

Заменяем $(-1)^{f\left(x_{l_{i}}, \ldots, x_{l_{i}+n-1}\right)}$ по формуле обратного преобразования Уолша-Адамара (теорема 1):

$$
(-1)^{f\left(x_{l_{i}}, \ldots, x_{l_{i}+n-1}\right)}=\frac{1}{2^{n}} \sum_{\left(\alpha^{(i, 0)}, \ldots, \alpha^{(i, n-1)}\right) \in V_{n}} W_{f}\left(\alpha^{(i, 0)}, \ldots, \alpha^{(i, n-1)}\right)(-1)^{\oplus_{s=0}^{n-1} \alpha^{(i, s)} x_{l_{i}+s}},
$$

затем перемножаем, и в выражении $(-1)^{\bigoplus_{i=1}^{m}} \bigoplus_{s=0}^{n-1} \alpha^{(i, s)} x_{l_{i}+s}$ приводим подобные члены следующим образом:

$$
\bigoplus_{i=1}^{m} \bigoplus_{s=0}^{n-1} \alpha^{(i, s)} x_{l_{i}+s}=\bigoplus_{k=1}^{N} x_{k} \bigoplus_{(i, s) \in J_{k}} \alpha^{(i, s)}
$$

Теперь меняем порядок суммирования по $x_{l_{i}+s}$ и $\alpha^{(i, s)}$, пользуясь соотношением

$$
\sum_{\left(x_{1}, \ldots, x_{N}\right) \in V_{N}}(-1)^{\oplus_{k=1}^{N} x_{k} \oplus_{(i, s) \in J_{k}} \alpha^{(i, s)}}= \begin{cases}2^{N}, & \text { если } \bigoplus_{(i, s) \in J_{k}} \alpha^{(i, s)}=0, k=1, \ldots, N, \\ 0 & \text { в противном случае }\end{cases}
$$

и приходим к требуемому условию (2).

Пример 1. Пусть $f \in \mathscr{F}_{4}$. Чтобы проверить, что функция $f$ является $\rho(3 ; 1,2,3)$-уравновешенной, необходимо и достаточно проверить, что выполнены равенства

$$
\begin{aligned}
& W_{f}(0,0,0,0)=0 \text {, } \\
& \sum_{\left(a^{(1)}, a^{(2)}, a^{(3)}\right) \in V_{3}} W_{f}\left(0, a^{(1)}, a^{(2)}, a^{(3)}\right) W_{f}\left(a^{(1)}, a^{(2)}, a^{(3)}, 0\right)=0 \text {, } \\
& \sum_{\left(a^{(1)}, a^{(2)}\right) \in V_{2}} W_{f}\left(0,0, a^{(1)}, a^{(2)}\right) W_{f}\left(a^{(1)}, a^{(2)}, 0,0\right)=0, \\
& \sum W_{f}\left(0, a^{(1)}, a^{(3)} \oplus a^{(4)}, a^{(5)} \oplus a^{(6)}\right) W_{f}\left(a^{(1)}, a^{(3)}, a^{(5)}, a^{(2)}\right) W_{f}\left(a^{(4)}, a^{(6)}, a^{(2)}, 0\right)=0 .
\end{aligned}
$$
$\left(a^{(1)}, \ldots, a^{(6)}\right) \in V_{6}$ 
Пример 2. Рассмотрим функцию $f\left(x_{1}, x_{2}, x_{3}, x_{4}\right)=x_{1} x_{3} \oplus x_{2} x_{3} \oplus x_{2} x_{3} x_{4} \oplus x_{4}$. Она является $\rho(2 ; 1,2)$-уравновешенной, так как для нее выполнены равенства $W_{f}(0,0,0,0)=0$ и

$$
\sum_{\left(a^{(1)}, a^{(2)}, a^{(3)}\right) \in V_{3}} W_{f}\left(0, a^{(1)}, a^{(2)}, a^{(3)}\right) \cdot W_{f}\left(a^{(1)}, a^{(2)}, a^{(3)}, 0\right)=0 .
$$

С другой стороны, так как выражение $\sum_{\left(a^{(1)}, a^{(2)}\right) \in V_{2}} W_{f}\left(0,0, a^{(1)}, a^{(2)}\right) \cdot W_{f}\left(a^{(1)}, a^{(2)}, 0,0\right)$ отлично от нуля, $\rho(2 ; 1,3)$-уравновешенной данная функция не является.

Суммируя полученные результаты, приходим к следующему утверждению.

Теорема 4. Функиия $f \in \mathscr{F}_{n}$ является совершенно уравновешенной тогда и только тогда, когда ее коэффициенты Уолиа-Адамара для любого натурального т и любого допустимого набора номеров аргументов для суммы (1) удовлетворяют соотношению (2).

Для фиксированного $n$ интересен вопрос, при каком наименьшем $M \rho_{M}$-уравновешенность булевой функции означает ее совершенную уравновешенность. В [3] доказано, что в качестве обладающего таким свойством $M$ можно выбрать $M=2^{n-2}\left(2^{n-1}-1\right)+n$.

В [1] Голичем было доказано следующее утверждение, из которого вытекает, что в качестве $M$ можно выбрать $M=n$.

Лемма 1 ([1]). Булева функция $\mathbf{f} \in \mathscr{F}_{n}$ совершенно уравновешена тогда и только тогда, когда она является $\rho_{n}$-уравновешенной.

В доказательстве утверждения в [1] содержался существенный пробел, однако опровержений данного утверждения до работы [2] не предлагалось.

Пример 3 ([2]). Рассмотрим следующую функцию $f \in \mathscr{F}_{5}$ :

$$
\begin{aligned}
& f\left(x_{1}, x_{2}, x_{3}, x_{4}, x_{5}\right) \\
& \quad=x_{2} \oplus x_{3} \oplus x_{5} \oplus x_{1} x_{4} \oplus x_{1} x_{5} \oplus x_{2} x_{4} \oplus x_{2} x_{5} \oplus x_{3} x_{5} \oplus x_{1} x_{4} x_{5} \oplus x_{2} x_{4} x_{5}
\end{aligned}
$$

Данная функция $\rho_{5}$-уравновешена и даже $\rho_{6}$-уравновешена, но не $\rho_{7}$-уравновешена, и следовательно, $f$ не является совершенно уравновешенной, что противоречит лемме Голича и даже ее ослабленному варианту (с условием на $\rho_{n+1}$-уравновешенность вместо $\rho_{n}$-уравновешенности).

Покажем, что ни для какого сколь угодно большого $c \in \mathbf{N}$ ослабленный вариант леммы Голича с условием на $\rho_{n+c}$-уравновешенность не является верным.

Лемма 2. Пусть $f \in \mathscr{F}_{n}$. Функциия

$$
f^{(k)}\left(x_{1}, x_{2}, \ldots, x_{k n-k+1}\right)=f\left(x_{1}, x_{k+1}, x_{2 k+1}, \ldots, x_{(n-1) k+1}\right)
$$

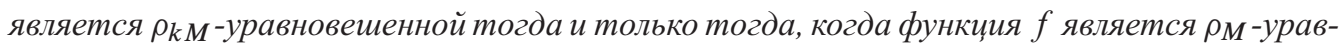
новешенной.

Доказательство. Необходимость. Пусть функция $f^{(k)}$ является $\rho_{k M}$-уравновешенной. Как нетрудно убедиться, для всякого набора $\left(y_{1}, y_{2}, \ldots, y_{M}\right) \in V_{M}$ верно равенство

$$
\left|f_{\rho_{k M}}^{(k)}{ }^{-1}(\underbrace{y_{1}, y_{1}, \ldots, y_{1}}_{k}, \underbrace{y_{2}, y_{2}, \ldots, y_{2}}_{k}, \ldots, \underbrace{y_{M}, y_{M}, \ldots, y_{M}}_{k})\right|=\left|f_{\rho_{M}}^{-1}\left(y_{1}, y_{2}, \ldots, y_{M}\right)\right|^{k} \text {. }
$$


Предположим противное: функция $f \in \mathscr{F}_{n}$ не является $\rho_{M}$-уравновешенной. Тогда существует пара наборов $\left(y_{1}^{\prime}, y_{2}^{\prime}, \ldots, y_{M}^{\prime}\right),\left(y_{1}^{\prime \prime}, y_{2}^{\prime \prime}, \ldots, y_{M}^{\prime \prime}\right) \in V_{M}$, для которых выполнено $\left|f_{\rho_{M}}^{-1}\left(y_{1}^{\prime}, y_{2}^{\prime}, \ldots, y_{M}^{\prime}\right)\right| \neq\left|f_{\rho_{M}}^{-1}\left(y_{1}^{\prime \prime}, y_{2}^{\prime \prime}, \ldots, y_{M}^{\prime \prime}\right)\right|$. Учитывая (4), получаем, что

$$
\begin{aligned}
\mid f_{\rho_{k M}}^{(k)}-1 & (\underbrace{y_{1}^{\prime}, y_{1}^{\prime}, \ldots, y_{1}^{\prime}}_{k}, \underbrace{y_{2}^{\prime}, y_{2}^{\prime}, \ldots, y_{2}^{\prime}}_{k}, \ldots, \underbrace{y_{M}^{\prime}, y_{M}^{\prime}, \ldots, y_{M}^{\prime}}_{k}) \mid \\
& \neq\left|f_{\rho_{k M}(k)}^{-1}(\underbrace{y_{1}^{\prime \prime}, y_{1}^{\prime \prime}, \ldots, y_{1}^{\prime \prime}}_{k}, \underbrace{y_{2}^{\prime \prime}, y_{2}^{\prime \prime}, \ldots, y_{2}^{\prime \prime}}_{k}, \ldots, \underbrace{y_{M}^{\prime \prime}, y_{M}^{\prime \prime}, \ldots, y_{M}^{\prime \prime}}_{k})\right| .
\end{aligned}
$$

Таким образом, функция $f^{(k)}$ не является $\rho_{k}$-уравновешенной, что противоречит предположению.

Достаточность. Для всякого набора $\left(y_{1}, y_{2}, \ldots, y_{k M}\right) \in V_{k M}$ верно равенство

$$
\begin{aligned}
& f_{\rho_{k M}}^{(k)}{ }^{-1}\left(y_{1}, y_{2}, \ldots, y_{k M}\right)=\left\{\left(x_{1}, x_{2}, \ldots, x_{k M+k(n-1)}\right) \in V_{k M+k(n-1)} \mid\right. \\
& \left.\quad\left(x_{i}, x_{k+i}, \ldots, x_{k(M+n-2)+i}\right) \in f_{\rho_{M}}^{-1}\left(y_{i}, y_{k+i}, \ldots, y_{k(M-1)+i}\right), i=1,2, \ldots, k\right\} .
\end{aligned}
$$

Пусть функция $f$ является $\rho_{M}$-уравновешенной. Тогда, как следует из (5), мощность множества $f_{\rho_{k M}}^{(k)}{ }^{-1}\left(y_{1}, y_{2}, \ldots, y_{k M}\right)$ не зависит от выбора $\left(y_{1}, y_{2}, \ldots, y_{k M}\right) \in V_{k M}$.

Следствие 3. Для любого $c \in \mathbf{N}$ существует $n \in \mathbf{N}$ и функици $f \in \mathscr{F}_{n}$, которая является

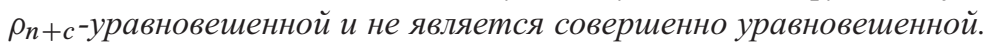

Доказательство. Зафиксируем произвольное $c \in \mathbf{N}$. Пусть $k=\lceil(c+1) / 2\rceil$. Рассмотрим функцию $f$ из примера 3 и соответствующую функцию $f^{(k)} \in \mathscr{F}_{4 k+1}$. Тогда $n=4 k+1$ и по лемме $2, f$ является $\rho_{6 k}$-уравновешенной, но не является $\rho_{7 k}$-уравновешенной. Следовательно, $f$ является $\rho_{n}+c$-уравновешенной, но не является совершенно уравновешенной.

\section{Список литературы}

1. Golić J., On the security of nonlinear filter generators. Lect. Notes Computer Sci. (1996) 1039, 173-188.

2. Smyshlyaev S. V., Perfectly balanced Boolean functions and Golić conjecture. J. Cryptology (2012) 25, №3, 464-483.

3. Бабаш А. В., Запреты автоматов и двоичных функций. Труды по дискретной математике (2006) 9, 7-20.

4. Логачев О. А., Сальников А. А., Ященко В. В., Булевы функиии в теории кодирования и криптологии. МЦНМО, Москва, 2004.

5. Логачев О. А., Смышляев С. В., Ященко В. В., Новые методы изучения совершенно уравновешенных булевых функций. Дискретная математика (2009) 21, №2, 51-74.

6. Смышляев С. В., Барьеры совершенно уравновешенных булевых функций. Дискретная математика (2010) 22, №2, 66-79.

7. Сумароков С. Н., Запреты двоичных функций и обратимость для одного класса кодирующих устройств. Обозрение прикладной и промышленной математики (1994) 1, №1, 33-55.

8. Ященко В. В., Свойства булевых отображений, сводимые к свойствам их координатных функций. Вестник МГУ, сер. матем. (1997), № 4 11-13. 